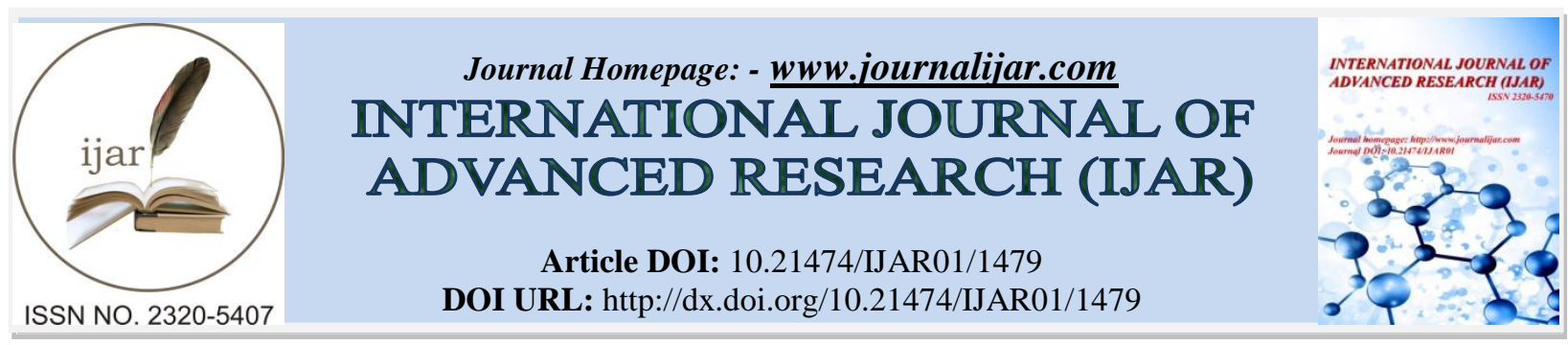

RESEARCH ARTICLE

\title{
EFFECT OF TEMPERATURE ON DEGRADATION POTENTIAL OF SELECTED BACTERIAL STRAINS FROM EFFLUENT TREATMENT PLANT OF COIR INDUSTRY.
}

\author{
*Sowparnika $\mathbf{S}^{1}$., Rajani $\mathbf{V}^{2}$. and Aswathy $\mathbf{R}^{3}$. \\ 1. Assistant Professor, P.G \& Research Department of Zoology and Biotechnolgy, Pachaiyappa's College, Chennai \\ -6000030 , India \\ 2. Research scholar, PG and Research Department of Advanced Zoology \& Biotechnology, Sir Theagaraya College, \\ Chennai, Tamilnadu, India \\ 3. Associate Professor, Department of Zoology, Unit of Aquaculture and Cryobiology, University of Madras, \\ Guindy campus, Chennai
}

\section{Manuscript Info}

Manuscript History

Received: 15 July 2016

Final Accepted: 13 August 2016

Published: September 2016

Key words:-

Brucella sp, Coir Industry, Industrial

effluent, Phenol degradation,

Temperature.

\section{Abstract}

Phenol-degrading bacteria exist widely in the environments and they are usually isolated from phenol contaminated site. Phenol and its components are majorly toxic to the environment. It is hazardous when exposed to the organisms' surface. The aim of the study was to isolate and identify the phenol degrading bacteria from effluent treatment plant of coir industry and to analyse the effect of temperature on the degradation potential. From the isolated bacterial strains, 5 most potent strains were selected for the study (Brucella sp, Aquaspirillum sp, Erwinia sp, Aeromonas sp and Moraxella sp). To determine the effect of temperature the experiments were carried out at different temperatures such as $27^{\circ} \mathrm{C}, 35^{\circ} \mathrm{C}$ and $47^{\circ} \mathrm{C}$. The selected bacterial strains were grown in medium with different phenol concentrations $(200,400,600,800$ and $1000 \mathrm{ppm})$. The growth of bacteria and phenol concentration in the media were observed for the three selected temperatures. The decrease in phenol concentration accompanied with increase in biomass. From the preliminary analysis, the most potent strains were found to be Brucella sp and Aeromonas sp. The medium with $800 \mathrm{ppm}$ phenol showed better degradation for all the selected strains. At the same time when the prescribed temperature was changed, the degradation potential was reduced and was unstable.

Copy Right, IJAR, 2016,. All rights reserved.

\section{Introduction:-}

Phenol and its derivatives are the most common toxic organic pollutant of various industrial wastewaters and therefore it requires proper treatment before being discharged (Shewtha et al., 2013). Phenol and phenolic compounds are of general use in many industries such as polymeric resin production and oil refining. As a result, these compounds are commonly encountered in industrial effluents and surface water. The low volatility of phenol and its affinity for water make oral consumption of contaminated water the greatest risk to humans(D. Hank et al., 2010) .Toxicity of phenolic compounds inhibits biological treatment or even eliminates sensitive micro-organisms from biological wastewater treatment process and significantly reduces the biodegradation of the other components 
(Yan et al., 2006). The presence of phenol in water imparts carbolic odour to receiving water bodies and can cause toxic effects on aquatic flora and fauna (Ghadhi and Sangodkar, 1995). Hence, it is necessary to remove phenol from industrial effluents before discharging them into the environment. Many technologies exist for the treatment of phenols, but their use is intermediates particularly from non biological process. Many aerobic bacteria have been confirmed to use aromatic carbon as a pure source of carbon and energy (Paller et al., 1995) which suggests using phenol as nutrient to the organism and thereby converts phenol to nontoxic component. Growth and biodegradation of any microorganism depends on various physiochemical parameters. Aim of this study was to optimize the temperature of incubation. Study on growth of bacteria at different temperature was carried out and the optimum temperature for the better biodegradation of phenol was found out.

\section{Materials and methods:-}

Samples from effluent treatment plant of paper industry were collected and serially diluted. Microbial enrichment was done using nutrient broth with different phenol concentrations (10, 20, 30, 40, 50 ppm). From the 50 ppm culture, organisms were collected and added to sorbitol agar medium with varying concentrations of phenol (200, 400, 600, 800 and $1000 \mathrm{ppm})$.

\section{Estimation of Total Phenol:-}

Estimation of total phenol was carried out the method of (Bray et al., 1954).

\section{Observation of Total Growth:-}

The growth rates of the microbes were observed by spectrophotometric analysis.

\section{Identification of Isolates:-}

Biochemical characterization was done based on Bergy's manual of Determinative Bacteriology (1994) and Cappuccino et al., (1999, 2000)

\section{Effect of Temperature on Growth and Total Phenol:-}

The effect of temperature on total growth and phenol degradation of the selected strains were studied.

\section{Results and Discussion:-}

Serially diluted effluent of coir industry was transferred to 10, 20, 30, 40 and 50 ppm phenol having minimal media. From the $50 \mathrm{ppm}$ medium, they were inoculated into selective sorbitol agar media with varying phenol concentrations $(200,400,600,800,1000 \mathrm{ppm})$. In this study, five most potent bacterial strains were isolated and identified as Brucella sp, Aquaspirillum sp, Erwinia sp, Aeromonas sp and Moraxella sp. They were further used to investigate the growth and total phenol content in varying phenol concentrations. They were also used to study the effect of temperature. To determine the effect of temperature the experiments were carried out at different temperatures such as $27^{\circ} \mathrm{C}, 35^{\circ} \mathrm{C}$ and $47^{\circ} \mathrm{C}$. The selected bacterial strains were grown in medium with different phenol concentrations (200, 400, 600, 800 and 1000ppm). Neumann et al., 2004, adapted Pseudomonas strains to high concentrations of phenol $(1000 \mathrm{mg} / \mathrm{L})$ and further biodegradation was carried out at a concentration of 800 $\mathrm{mg} / \mathrm{L}$. The total phenol and growth of selected bacterial strains, during different temperatures $\left(27^{\circ} \mathrm{C}, 35^{\circ} \mathrm{C}\right.$ and $\left.47^{\circ}\right)$ are given in the tables 1 to 15 .

At $27^{\circ} \mathrm{C}$ ( tables 1 to 5), phenol degradation in $200 \mathrm{ppm}$ concentration was detected to be highest at 48 hrs of incubation by Aeromonas sp (0.0191) then decresed by Brucella sp (0.0376), Erwinia sp (0.0411) ,the least degradations were showed by Aquaspirillum sp and Moraxella sp (0.0465). The growth rates of the bacterial strain that was noted to be the highest was at $24 \mathrm{hrs}$ of incubation by Aeromonas sp (0.0349) followed by Aquaspirillum $\mathrm{sp}(0.0269)$,Erwinia $\mathrm{sp}(0.0251)$, Brucella $\mathrm{sp}(0.0188)$ and Moraxella $\mathrm{sp}(0.0154)$. In 400ppm concentration ,the highest degradation was at $72 \mathrm{hrs}$ of incubation by Aeromonas sp (0.0207) followed by Erwinia sp (0.0246), Aquaspirillum sp (0.0292), Moraxella sp (0.0302) and Brucella sp (0.0356). The growth rate was maximum for Aeromonas sp at $24 \mathrm{hrs}$ of incubation (0.0300), then Aquaspirillum sp (0.0269), Erwinia sp (0.0251), Brucella sp (0.0188) and Moraxella $\mathrm{sp}(0.0154)$.

Table 1:- Total phenol and growth of coir effluent sample 200ppm

\begin{tabular}{|l|l|l|l|l|l|l|l|l|}
\hline \multirow{2}{*}{ Strains } & \multicolumn{3}{|c|}{ Total Phenol (720nm) } & \multicolumn{4}{c|}{ Growth (600nm) } \\
\cline { 2 - 9 } & 24 & 48 & 72 & 96 & 24 & 48 & 72 & 96 \\
\hline
\end{tabular}




\begin{tabular}{|l|l|l|l|l|l|l|l|l|}
\hline Brucella $\mathrm{sp}$ & 0.0960 & 0.0376 & 0.0452 & 0.0258 & 0.0188 & 0.0103 & 0.0279 & 0.0104 \\
\hline Aquaspirillum sp & 0.0444 & 0.0465 & 0.0393 & 0.0298 & 0.0269 & 0.0122 & 0.0098 & 0.0092 \\
\hline Erwinia $\mathrm{sp}$ & 0.0856 & 0.0411 & 0.0305 & 0.0407 & 0.0251 & 0.0110 & 0.0084 & 0.0101 \\
\hline Aeromonas $\mathrm{sp}$ & 0.0781 & 0.0191 & 0.0275 & 0.0313 & 0.0349 & 0.0104 & 0.0090 & 0.0105 \\
\hline Moraxella $\mathrm{sp}$ & 0.0377 & 0.0465 & 0.0289 & 0.0350 & 0.0154 & 0.0132 & 0.0110 & 0.0095 \\
\hline
\end{tabular}

Table 2:- Total phenol and growth of coir effluent sample 400ppm

\begin{tabular}{|l|l|l|l|l|l|l|l|l|}
\hline \multirow{2}{*}{ Strains } & \multicolumn{3}{|c|}{ Total } & Phenol (720nm) & \multicolumn{3}{c|}{ Growth (600nm) } \\
\cline { 2 - 9 } & 24 & 48 & 72 & 96 & 24 & 48 & 72 & 96 \\
\hline Brucella $\mathrm{sp}$ & 0.0278 & 0.0359 & 0.0356 & 0.0309 & 0.0188 & 0.0286 & 0.0097 & 0.0058 \\
\hline Aquaspirillum $\mathrm{sp}$ & 0.0354 & 0.0503 & 0.0292 & 0.0403 & 0.0269 & 0.0144 & 0.0103 & 0.0094 \\
\hline Erwinia $\mathrm{sp}$ & 0.0669 & 0.0308 & 0.0246 & 0.0264 & 0.0251 & 0.0213 & 0.0100 & 0.0088 \\
\hline Aeromonas $\mathrm{sp}$ & 0.0349 & 0.0410 & 0.0207 & 0.0265 & 0.0300 & 0.0242 & 0.0106 & 0.0080 \\
\hline Moraxella $\mathrm{sp}$ & 0.0269 & 0.0498 & 0.0302 & 0.0213 & 0.0154 & 0.0233 & 0.0099 & 0.0090 \\
\hline
\end{tabular}

Table 3:- Total phenol and growth of coir effluent sample 600ppm

\begin{tabular}{|l|l|l|l|l|l|l|l|l|}
\hline \multirow{2}{*}{ Strains } & \multicolumn{3}{|c|}{ Total } & Phenol (720nm) & \multicolumn{3}{c|}{ Growth (600nm) } \\
\cline { 2 - 9 } & 24 & 48 & 72 & 96 & 24 & 48 & 72 & 96 \\
\hline Brucella sp & 0.0393 & 0.0493 & 0.0344 & 0.0253 & 0.0075 & 0.0133 & 0.0112 & 0.0096 \\
\hline Aquaspirillum $\mathrm{sp}$ & 0.0600 & 0.0271 & 0.0361 & 0.0183 & 0.0060 & 0.0083 & 0.0104 & 0.0063 \\
\hline Erwinia sp & 0.0272 & 0.0319 & 0.0279 & 0.0238 & 0.0071 & 0.0137 & 0.0074 & 0.0076 \\
\hline Aeromonas $\mathrm{sp}$ & 0.0454 & 0.0190 & 0.0349 & 0.0224 & 0.0054 & 0.0106 & 0.0089 & 0.0092 \\
\hline Moraxella $\mathrm{sp}$ & 0.0282 & 0.0297 & 0.0289 & 0.0184 & 0.0066 & 0.0082 & 0.0102 & 0.0084 \\
\hline
\end{tabular}

Table 4:- Total phenol and growth of coir effluent sample 800ppm

\begin{tabular}{|l|l|l|l|l|l|l|l|l|}
\hline \multirow{2}{*}{ Strains } & \multicolumn{4}{|c|}{ Total Phenol (720nm) } & \multicolumn{3}{c|}{ Growth (600nm) } \\
\cline { 2 - 10 } & 24 & 48 & 72 & 96 & 24 & 48 & 72 & 96 \\
\hline Brucella $\mathrm{sp}$ & 0.0356 & 0.0507 & 0.0365 & 0.0340 & 0.0188 & 0.0101 & 0.0132 & 0.0080 \\
\hline Aquaspirillum $\mathrm{sp}$ & 0.0326 & 0.0257 & 0.0284 & 0.0333 & 0.0269 & 0.0103 & 0.0093 & 0.0079 \\
\hline Erwinia $\mathrm{sp}$ & 0.0264 & 0.0307 & 0.0406 & 0.0280 & 0.0251 & 0.0079 & 0.0103 & 0.0083 \\
\hline Aeromonas $\mathrm{sp}$ & 0.0367 & 0.0442 & 0.0355 & 0.0238 & 0.0349 & 0.0100 & 0.0112 & 0.0079 \\
\hline Moraxella $\mathrm{sp}$ & 0.0312 & 0.0220 & 0.0267 & 0.0168 & 0.0154 & 0.0139 & 0.0104 & 0.0091 \\
\hline
\end{tabular}

Table 5:- Total phenol and growth of coir effluent sample 1000ppm

\begin{tabular}{|l|l|l|l|l|l|l|l|l|}
\hline \multirow{2}{*}{ Strains } & \multicolumn{3}{|c}{ Total } & \multicolumn{4}{c|}{ Ghenol (720nm) } & \multicolumn{3}{c|}{ Growth (600nm) } \\
\cline { 2 - 9 } & 24 & 48 & 72 & 96 & 24 & 48 & 72 & 96 \\
\hline Brucella $\mathrm{sp}$ & 0.0185 & 0.0379 & 0.0389 & 0.0358 & 0.0055 & 0.0127 & 0.0090 & 0.0119 \\
\hline Aquaspirillum $\mathrm{sp}$ & 0.0269 & 0.0374 & 0.0378 & 0.0332 & 0.0052 & 0.0124 & 0.0110 & 0.0096 \\
\hline Erwinia $\mathrm{sp}$ & 0.0281 & 0.0433 & 0.0377 & 0.0170 & 0.0067 & 0.0249 & 0.0083 & 0.0117 \\
\hline Aeromonas $\mathrm{sp}$ & 0.0201 & 0.0456 & 0.0303 & 0.0300 & 0.0060 & 0.0111 & 0.0100 & 0.0098 \\
\hline Moraxella $\mathrm{sp}$ & 0.0308 & 0.0332 & 0.0293 & 0.0275 & 0.0062 & 0.0116 & 0.0098 & 0.0101 \\
\hline
\end{tabular}

The phenol degradation at 600ppm was highest during $96 \mathrm{hrs}$ of incubation by Aquaspirillum sp (0.0183) followed by Moraxella sp (0.0184), Aeromonas sp (0.0224), Erwinia sp (0.0238) and Brucella sp (0.0253). Growth rate was noted the highest for Erwinia sp at $48 \mathrm{hrs}$ of incubation (0.0137) and followed by Brucella sp (0.0133), Aeromonas sp (0.0106), Aquaspirillum sp (0.0083), and Moraxella sp (0.0082) .

In 800ppm the phenol degradation was noted highest for Moraxella sp (0.0168) at 96 hrs of incubation and the decreased by, Aeromonas sp (0.0238), Erwinia sp (0.0280), Aquaspirillum sp(0.0333), and the Brucella sp (0.0350).Growth rate was highest for Aeromonas sp at $24 \mathrm{hrs}$ of incubation (0.0349)and then Aquaspirillum sp (0.0269), Erwinia sp(0.0251), Brucella sp (0.0188), and Moraxella sp (0.0154) . 
In 1000ppm concentration the highest phenol degradation was obtained by Erwinia sp (0.0170) at 96 hrs of incubation and then followed by Moraxella sp (0.0275), Aeromonas sp (0.0300), Aquaspirillum sp (0.0332), and Brucella sp (0.0358). Growth rate was the highest for Erwinia sp at 48 hrs of incubation (0.0249) and then decreased with Brucella sp (0.0127), Aquaspirillum sp (0.0124), Moraxella sp (0.0116), and Aeromonas sp (0.0111) .

At $35^{\circ} \mathbf{C}$ ( tables 6 to 10), in $200 \mathrm{ppm}$ medium, it was observed that Brucella sp showed highest phenol degradation at $48 \mathrm{hrs} \mathrm{hrs} \mathrm{(0.0057)} \mathrm{followed} \mathrm{by} \mathrm{Aquaspirillum} \mathrm{sp} \mathrm{(0.0119),} \mathrm{Erwinia} \mathrm{sp} \mathrm{(0.0205),} \mathrm{Aeromonas} \mathrm{sp} \mathrm{(0.0132)} \mathrm{and}$ Moraxella sp (0.0162).The least degradation was showed by Aeromonas sp for $24 \mathrm{hrs}(0.0880)$ incubation. The growth rate was observed highest for Aquaspirillum sp at $72 \mathrm{hrs}$ of incubation (0.0817) followed by Aeromonas sp (0.0777), Brucella sp (0.0768), Moraxella sp (0.0757) and Erwinia sp (0.0725).

Total phenol and growth at $35^{\circ} \mathrm{C}$

Table 6:- Total phenol and growth of coir effluent sample (200ppm)

\begin{tabular}{|l|l|l|l|l|l|l|l|l|}
\hline \multirow{2}{*}{ Strains } & \multicolumn{4}{|l|}{ Total Phenol $(720 \mathrm{~nm})$} & \multicolumn{4}{l|}{ Growth $(600 \mathrm{~nm})$} \\
\cline { 2 - 10 } & 24 & 48 & 72 & 96 & 24 & 48 & 72 & 96 \\
\hline Brucella $\mathrm{sp}$ & 0.0144 & 0.0057 & 0.0220 & 0.0101 & 0.0631 & 0.0063 & 0.0768 & 0.0708 \\
\hline Aquaspirillum $\mathrm{sp}$ & 0.0461 & 0.0119 & 0.0190 & 0.0086 & 0.0634 & 0.0072 & 0.0817 & 0.0752 \\
\hline Erwinia $\mathrm{sp}$ & 0.0687 & 0.0205 & 0.0153 & 0.0094 & 0.0630 & 0.0068 & 0.0725 & 0.0618 \\
\hline Aeromonas $\mathrm{sp}$ & 0.0880 & 0.0132 & 0.0265 & 0.0153 & 0.0579 & 0.0054 & 0.0777 & 0.0739 \\
\hline Moraxella $\mathrm{sp}$ & 0.0737 & 0.0162 & 0.0206 & 0.0135 & 0.0540 & 0.0051 & 0.0757 & 0.0755 \\
\hline
\end{tabular}

Table 7:- Total phenol and growth of coir effluent sample (400ppm)

\begin{tabular}{|l|l|l|l|l|l|l|l|l|}
\hline \multirow{2}{*}{ Strains } & \multicolumn{4}{l}{ Total Phenol $(720 \mathrm{~nm})$} & \multicolumn{4}{l|}{ Growth (600nm) } \\
\cline { 2 - 9 } & 24 & 48 & 72 & 96 & 24 & 48 & 72 & 96 \\
\hline Brucella $\mathrm{sp}$ & 0.0647 & 0.0139 & 0.0168 & 0.0087 & 0.0477 & 0.0064 & 0.0658 & 0.0324 \\
\hline Aquaspirillum $\mathrm{sp}$ & 0.0482 & 0.0103 & 0.0144 & 0.0093 & 0.0576 & 0.0067 & 0.0744 & 0.0694 \\
\hline Erwinia $\mathrm{sp}$ & 0.0863 & 0.0100 & 0.0218 & 0.0108 & 0.0598 & 0.0056 & 0.0713 & 0.0329 \\
\hline Aeromonas $\mathrm{sp}$ & 0.0888 & 0.0065 & 0.0178 & 0.0134 & 0.0587 & 0.0054 & 0.0754 & 0.0355 \\
\hline Moraxella $\mathrm{sp}$ & 0.0067 & 0.0052 & 0.0277 & 0.0120 & 0.0623 & 0.0051 & 0.0709 & 0.0574 \\
\hline
\end{tabular}

Table 8:- Total phenol and growth of coir effluent sample (600ppm)

\begin{tabular}{|l|l|l|l|l|l|l|l|l|}
\hline \multirow{2}{*}{ Strains } & \multicolumn{2}{|l|}{ Total Phenol (720nm) } & Growth (600nm) \\
\cline { 2 - 9 } & 24 & 48 & 72 & 96 & 24 & 48 & 72 & 96 \\
\hline Brucella $\mathrm{sp}$ & 0.0772 & 0.0170 & 0.0181 & 0.0106 & 0.0534 & 0.0083 & 0.0755 & 0.0672 \\
\hline Aquaspirillum $\mathrm{sp}$ & 0.0194 & 0.0021 & 0.0169 & 0.0132 & 0.0613 & 0.0079 & 0.0774 & 0.0706 \\
\hline Erwinia $\mathrm{sp}$ & 0.0832 & 0.0126 & 0.0259 & 0.0172 & 0.0554 & 0.0065 & 0.0680 & 0.0673 \\
\hline Aeromonas $\mathrm{sp}$ & 0.0702 & 0.0089 & 0.0209 & 0.0078 & 0.0582 & 0.0078 & 0.0688 & 0.0488 \\
\hline Moraxella $\mathrm{sp}$ & 0.0507 & 0.0093 & 0.0096 & 0.0080 & 0.0566 & 0.0090 & 0.0694 & 0.0434 \\
\hline
\end{tabular}

Table 9:- Total phenol and growth of coir effluent sample (800ppm)

\begin{tabular}{|l|l|l|l|l|l|l|l|l|}
\hline \multirow{2}{*}{ Strains } & \multicolumn{4}{|l}{ Total Phenol (720nm) } & \multicolumn{4}{l|}{ Growth $(600 \mathrm{~nm})$} \\
\cline { 2 - 10 } & 24 & 48 & 72 & 96 & 24 & 48 & 72 & 96 \\
\hline Brucella $\mathrm{sp}$ & 0.0971 & 0.0108 & 0.0250 & 0.0111 & 0.0577 & 0.0051 & 0.0734 & 0.0681 \\
\hline Aquaspirillum $\mathrm{sp}$ & 0.0314 & 0.0153 & 0.0230 & 0.0103 & 0.0574 & 0.0080 & 0.0740 & 0.0630 \\
\hline Erwinia $\mathrm{sp}$ & 0.0163 & 0.0092 & 0.0187 & 0.0124 & 0.0557 & 0.0052 & 0.0833 & 0.0762 \\
\hline Aeromonas $\mathrm{sp}$ & 0.0890 & 0.0226 & 0.0248 & 0.0192 & 0.0561 & 0.0065 & 0.0686 & 0.0647 \\
\hline Moraxella $\mathrm{sp}$ & 0.0820 & 0.0230 & 0.0188 & 0.0140 & 0.0555 & 0.0058 & 0.0715 & 0.0488 \\
\hline
\end{tabular}

\begin{tabular}{|l|l|l|l|l|l|l|l|l|}
\hline \multirow{2}{*}{ Strains } & \multicolumn{4}{|l|}{ Total Phenol $(720 \mathrm{~nm})$} & \multicolumn{4}{l|}{ Growth $(600 \mathrm{~nm})$} \\
\cline { 2 - 9 } & 24 & 48 & 72 & 96 & 24 & 48 & 72 & 96 \\
\hline Brucella $\mathrm{sp}$ & 0.0923 & 0.0140 & 0.0149 & 0.0091 & 0.0554 & 0.0076 & 0.0718 & 0.0694 \\
\hline
\end{tabular}




\begin{tabular}{|l|l|l|l|l|l|l|l|l|}
\hline Aquaspirillum $\mathrm{sp}$ & 0.0233 & 0.0077 & 0.0188 & 0.0076 & 0.0559 & 0.0084 & 0.0592 & 0.0465 \\
\hline Erwinia $\mathrm{sp}$ & 0.0563 & 0.0063 & 0.0333 & 0.0061 & 0.0552 & 0.0070 & 0.0649 & 0.0677 \\
\hline Aeromonas $\mathrm{sp}$ & 0.0572 & 0.0069 & 0.0200 & 0.0064 & 0.0549 & 0.0072 & 0.0640 & 0.0631 \\
\hline Moraxella $\mathrm{sp}$ & 0.0652 & 0.0134 & 0.0190 & 0.0106 & 0.0594 & 0.0060 & 0.0738 & 0.0434 \\
\hline
\end{tabular}

Table 10:-Total phenol and growth of coir effluent sample (1000ppm)

In 400ppm medium Moraxella sp showed highest phenol degradation at $48 \mathrm{hrs}(0.0052)$ followed by Aeromonas sp (0.0065), Erwinia sp (0.0100), Aquaspirillum $\mathrm{sp}(0.0103)$ and Brucella $\mathrm{sp}(0.0139)$.The least degradation was observed for Aeromonas sp (0.0888) at $24 \mathrm{hrs}$ incubation. The growth rate was observed highest for Aquaspirillum sp at $72 \mathrm{hrs}$ of incubation (0.0817) followed by Aeromonas sp (0.0777), Brucella sp (0.0768), Moraxella sp (0.0757) and Erwinia sp (0.0725) .

In 600ppm medium, Aquaspirillum sp showed highest phenol degradation at $48 \mathrm{hrs}$ incubation (0.0021) followed by Aeromonas sp (0.0089), Moraxella sp(0.0093), Erwinia sp (0.0126) and Brucella sp( 0.0170). The growth rate was observed to be highest for Aquaspirillum sp at $72 \mathrm{hrs}$ of observation (0.0817) followed by Aeromonas sp (0.0777), Brucella sp (0.0768), Moraxella sp (0.0757) and Erwinia sp (0.0725) .

In 800ppm medium, Erwinia sp showed highest phenol degradation at 48hrs incubation (0.0092) followed by Brucella sp (0.0108), Aquaspirillum (0.0153), Aeromonas sp (0.0226), and Moraxella sp (0.0230). The least phenol degradation was shown by Brucella sp (0.0971) at $24 \mathrm{hrs}$ of incubation. The growth rate was observed to be highest for Aquaspirillum sp at $72 \mathrm{hrs}$ of incubation (0.0817) followed by Aeromonas sp (0.0777), Brucella sp (0.0768), Moraxella sp (0.0757) and Erwinia sp (0.0725) . Shen and Wang (1993) conducted experiments on simultaneous chromium reduction and phenol degradation in a co culture of Escherium coli and Pseudomonas putida. They also reported similar growth pattern with a short lag period, followed by an exponential growth phase and then a declining growth phase.

In 1000ppm medium the phenol degradation rate was highest for Erwina sp (0.0061) followed by Aeromonas sp (0.0064), Aquaspirillum sp (0.0076) Brucella sp (0.0091) and Moraxella sp (0.0106) . The growth rate was observed highest for Moraxella sp at $72 \mathrm{hrs}$ of incubation (0.0738) followed by Brucella sp (0.0718), Aquaspirillum sp (0.0592), Erwinia sp (0.0649) and Aeromonas sp (0.0640). Mrs. C H Supriya and Devaneehar, 2014 carried out a study to determine the effect of temperature the experiments were carried out at different temperatures such as $25^{\circ} \mathrm{C}, 35^{\circ} \mathrm{C}, 40^{\circ} \mathrm{C}, 50^{\circ} \mathrm{C}$ and $60^{\circ} \mathrm{C}$ for $120 \mathrm{hrs}$ of incubation period. The data showed that there was maximum phenol degradation takes place at room temperature of $35^{\circ} \mathrm{C}$ and on further increase in temperature. The rate of biodegradation decreases because the catalytic activity of the enzymes starts to decrease beyond that temperature. So, the optimum temperature for the maximum enzymatic activity is $35^{\circ} \mathrm{C}$.

At $47^{\circ} \mathbf{C}$ ( tables 11 to 15 ), 200ppm concentration of phenol showed highest degradation at 24 hrs of incubation by Brucella sp (0.0114) followed by Moraxella sp (0.0246), Erwinia sp (0.0373), Aquaspirillum sp (0.0403), and Aeromonas sp (0.0570). The growth rate was indicated to be highest at $48 \mathrm{hrs}$ of incubation by Aeromonas sp (0.0563), then decreased by Aquaspirillum sp (0.0470), Brucella sp (0.0392), Erwinia sp (0.0314), and Moraxella sp (0.0292) .

Total phenol and growth at $47^{\circ} \mathrm{C}$

Table 11:- Total phenol and growth of coir effluent sample $200 \mathrm{ppm}$

\begin{tabular}{|l|l|l|l|l|l|l|l|l|}
\hline \multirow{2}{*}{ Strains } & \multicolumn{3}{|c|}{ Total } & Phenol (720nm) & \multicolumn{3}{c|}{ Growth (600nm) } \\
\cline { 2 - 10 } & 24 & 48 & 72 & 96 & 24 & 48 & 72 & 96 \\
\hline Brucella $\mathrm{sp}$ & 0.0117 & 0.0140 & 0.0350 & 0.0416 & 0.0209 & 0.0392 & 0.0222 & 0.0094 \\
\hline Aquaspirillum $\mathrm{sp}$ & 0.0403 & 0.0202 & 0.0438 & 0.0344 & 0.0185 & 0.0470 & 0.0126 & 0.0120 \\
\hline Erwinia $\mathrm{sp}$ & 0.0373 & 0.0372 & 0.0435 & 0.0315 & 0.0119 & 0.0314 & 0.0153 & 0.0092 \\
\hline Aeromonas $\mathrm{sp}$ & 0.0570 & 0.0368 & 0.0332 & 0.0301 & 0.0062 & 0.0563 & 0.0222 & 0.0125 \\
\hline Moraxella $\mathrm{sp}$ & 0.0246 & 0.0350 & 0.0457 & 0.0404 & 0.0077 & 0.0292 & 0.0175 & 0.0157 \\
\hline
\end{tabular}

Table 12:- Total phenol and growth of coir effluent sample 400ppm

\begin{tabular}{|l|l|l|}
\hline Strains & Total Phenol (720nm) & Growth (600nm) \\
\hline
\end{tabular}




\begin{tabular}{|l|l|l|l|l|l|l|l|l|}
\hline & 24 & 48 & 72 & 96 & 24 & 48 & 72 & 96 \\
\hline Brucella $\mathrm{sp}$ & 0.0250 & 0.0308 & 0.0656 & 0.0314 & 0.0129 & 0.0309 & 0.0280 & 0.0135 \\
\hline Aquaspirillum $\mathrm{sp}$ & 0.0336 & 0.0260 & 0.0485 & 0.0194 & 0.0203 & 0.0316 & 0.0105 & 0.0116 \\
\hline Erwinia $\mathrm{sp}$ & 0.0346 & 0.0304 & 0.0341 & 0.0180 & 0.0154 & 0.0334 & 0.0145 & 0.0118 \\
\hline Aeromonas $\mathrm{sp}$ & 0.0518 & 0.0319 & 0.0540 & 0.0361 & 0.0109 & 0.0193 & 0.0104 & 0.0142 \\
\hline Moraxella $\mathrm{sp}$ & 0.0223 & 0.0370 & 0.0441 & 0.0316 & 0.0135 & 0.0206 & 0.0111 & 0.0179 \\
\hline
\end{tabular}

Table 13 - Total phenol and growth of coir effluent sample 600ppm

\begin{tabular}{|l|l|l|l|l|l|l|l|l|}
\hline \multirow{2}{*}{ Strains } & \multicolumn{3}{|c|}{ Total Phenol (720nm) } & \multicolumn{3}{c|}{ Growth (600nm) } \\
\cline { 2 - 9 } & 24 & 48 & 72 & 96 & 24 & 48 & 72 & 96 \\
\hline Brucella $\mathrm{sp}$ & 0.0276 & 0.0272 & 0.0252 & 0.0248 & 0.0247 & 0.0157 & 0.0179 & 0.0229 \\
\hline Aquaspirillum $\mathrm{sp}$ & 0.0499 & 0.0298 & 0.0329 & 0.0346 & 0.0084 & 0.0121 & 0.0113 & 0.0182 \\
\hline Erwinia $\mathrm{sp}$ & 0.0485 & 0.0255 & 0.0398 & 0.0217 & 0.0086 & 0.0162 & 0.0067 & 0.0109 \\
\hline Aeromonas $\mathrm{sp}$ & 0.0238 & 0.0262 & 0.0378 & 0.0275 & 0.0125 & 0.0113 & 0.0128 & 0.0112 \\
\hline Moraxella $\mathrm{sp}$ & 0.0215 & 0.0363 & 0.0278 & 0.0322 & 0.0066 & 0.0096 & 0.0063 & 0.0131 \\
\hline
\end{tabular}

Table 14:- Total phenol and growth of coir effluent sample 800ppm

\begin{tabular}{|l|l|l|l|l|l|l|l|l|}
\hline \multirow{2}{*}{ Strains } & \multicolumn{3}{|c|}{ Total } & Phenol (720nm) & \multicolumn{3}{c|}{ Growth (600nm) } \\
\cline { 2 - 9 } & 24 & 48 & 72 & 96 & 24 & 48 & 72 & 96 \\
\hline Brucella $\mathrm{sp}$ & 0.0523 & 0.0314 & 0.0500 & 0.0417 & 0.0181 & 0.0472 & 0.0115 & 0.0134 \\
\hline Aquaspirillum $\mathrm{sp}$ & 0.0569 & 0.0372 & 0.0445 & 0.0338 & 0.0055 & 0.0284 & 0.0112 & 0.0124 \\
\hline Erwinia $\mathrm{sp}$ & 0.0453 & 0.0506 & 0.0600 & 0.0365 & 0.0059 & 0.0127 & 0.0113 & 0.0097 \\
\hline Aeromonas $\mathrm{sp}$ & 0.0276 & 0.0444 & 0.0383 & 0.0415 & 0.0064 & 0.0133 & 0.0079 & 0.0096 \\
\hline Moraxella $\mathrm{sp}$ & 0.0733 & 0.0428 & 0.0729 & 0.0427 & 0.0090 & 0.0291 & 0.0135 & 0.0145 \\
\hline
\end{tabular}

Table 15:-Total phenol and growth of coir effluent sample 1000ppm

\begin{tabular}{|l|l|l|l|l|l|l|l|l|}
\hline \multirow{2}{*}{ Strains } & \multicolumn{3}{|c|}{ Total Phenol (720nm) } & \multicolumn{3}{c|}{ Growth (600nm) } \\
\cline { 2 - 10 } & 24 & 48 & 72 & 96 & 24 & 48 & 72 & 96 \\
\hline Brucella $\mathrm{sp}$ & 0.0464 & 0.0264 & 0.0421 & 0.0415 & 0.0130 & 0.0104 & 0.0087 & 0.0122 \\
\hline Aquaspirillum $\mathrm{sp}$ & 0.0298 & 0.0268 & 0.0423 & 0.0159 & 0.0070 & 0.0148 & 0.0077 & 0.0034 \\
\hline Erwinia $\mathrm{sp}$ & 0.0439 & 0.0324 & 0.0374 & 0.0418 & 0.0115 & 0.0201 & 0.0084 & 0.0134 \\
\hline Aeromonas $\mathrm{sp}$ & 0.0220 & 0.0350 & 0.0378 & 0.0285 & 0.0071 & 0.0160 & 0.0076 & 0.0129 \\
\hline Moraxella $\mathrm{sp}$ & 0.0538 & 0.0242 & 0.0395 & 0.0433 & 0.0084 & 0.0184 & 0.0129 & 0.0144 \\
\hline
\end{tabular}

In 400ppm highest degradation was showed at $96 \mathrm{hrs}$ of incubation by Erwinia sp (0.0180) followed by Aquaspirillum sp (0.0194), Brucella sp (0.0314), Moraxella sp (0.0316) , and Aeromonas sp (0.0361).The growth rate was maximum for Erwinia sp at $48 \mathrm{hrs}$ of incubation (0.0334), then Aquaspirillum sp (0.0316), Brucella sp (0.0309), Moraxella sp (0.0206), and Aeromonas sp (0.0193).

The phenol degradation at 600ppm concentration was greatest at $24 \mathrm{hrs}$ of incubation by Moraxella sp (0.0215) followed by, Aeromonas sp (0.0238) , Brucella sp (0.0276), Erwinia sp (0.0485) and Aquaspirillum sp (0.0499).Growth rate was noted highest for Brucella sp at $24 \mathrm{hrs}$ of incubation (0.0247) and then Aeromonas sp (0.0125) ,Erwinia sp(0.0086) Aquaspirillum sp (0.0084), and Moraxella sp (0.0066) .

In 800ppm the phenol degradation was noted highest for Aeromonas sp (0.0276) at $24 \mathrm{hrs}$ of incubation and the decreased by , Erwinia sp (0.0453), Brucella sp (0.0523), Aquaspirillum $\mathrm{sp}(0.0569)$, and Moraxella sp (0.0733). Growth rate was noted to be highest for Brucella sp at 48 hrs of incubation (0.0472)and then Moraxella sp (0.0291), Aquaspirillum sp (0.0284), Aeromonas sp (0.0133), and Erwinia sp (0.0127).

In 1000ppm the highest phenol degradation was obtained by Aeromonas sp (0.0220) at $24 \mathrm{hrs}$ of incubation and then followed by Aquaspirillum sp (0.0298), Erwinia sp (0.0439) Brucella sp (0.0464), and Moraxella sp (0.0538). Highest growth rate was obtained by Erwinia sp at $48 \mathrm{hrs}$ of incubation (0.0201) and the decreased by Moraxella sp (0.0184), Aeromonas sp (0.0160) Aquaspirillum sp (0.0148) and Brucella sp (0.0104) . 
From this preliminary analysis, it was clear that the maximum degradation potential can be achieved only during the optimum temperature. When there occurs a change in temperature of incubation, the growth of strains as well as their degradation potential will be unstable.

\section{Conclusion:-}

Biodegradation is one of the cheapest methods with no production of hazardous by-products. This method is generally preferred due to lower costs and possibility of complete mineralization. The growth and phenol biodegradation study was carried out in sorbitol agar media with varying phenol concentrations. The effect of temperature on the rate of phenol degradation by the selected strains was carried out. It was observed that the rate of phenol biodegradation was significantly affected by temperature of incubation. From this, it was clear that when temperature is altered, growth also decreases and the degradation do not occur properly. Highly acclimatisable microbes are those which are able to degrade phenol at high concentration and at greatest rate will be the best phenol degrader candidates. The results of study on effect temperature revealed that the standard optimum temperature $\left(35^{\circ} \mathrm{C}\right)$ was required for normal bacterial growth and phenol consumption as their energy source. Future studies should be carried out to isolate more potent useful microbes and can be used for the waste management.

\section{Acknowledgement:-}

The authors are gratefully acknowledging the financial support from the University Grants Commission (UGC), for this study in the form of Minor Research Project.

\section{Reference:-}

1. Annadurai G, Rajesh Babu S, Mahesh KPO, Murugesant T (2000): Adsorption and biodegradation ofphenol by chitosan-immobilized Pseudomonas putida (NICM 2174). Bio process Eng. 22, 2000; 493-501.

2. Bray HG, Thorpe WV(1954) : Analysis of phenolic compounds of interest in metabolism." Meth. Biochem. Anal., 1, 1954, 27-52.

3. Cappuccino, JG, Sherman, N (1992) : Biochemical activities of microorganisms. In: Microbiology, A Laboratory Manual. The Benjamin / Cummings Publishing Co. California, USA.

4. D. Hank, N. Saidani, A. Namane and A. Hellal (2010) : Batch phenol biodegradation study and application of factorial experimental design,Journal of Engineering Science and Technology Review 3 (1) 123-127

5. Ghadi, S.C., Sangodkar, (1995) : Potentials of Pseudomonas cepacia PAA bioremediation of aquatic wastes containing phenol. Frontiers in Applied Environmental Microbiology (Ed.) A.

6. Mrs.CH. Supriya and DevaNeehar(2014): Biodegradation of phenol by Aspergillus niger. IOSR Journal Of Pharmacy Volume 4, Issue 7 (July 2014), PP. 11-17 11

7. Paller,G., Hommel, R.K and Kleber,H.P.,(1995):Phenol degradation by Acinetobacter calcoaceticus NCIB 8250. J. Basic Microbial. 35 : 325-335.

8. Robertson, B.K. and Alexander, M. (1992): Influence of calcium iron and pH on phosphate availability for microbial mineralization of organic chemicals. Appl. Environ. Microbiol. 58:38-41.

9. Shweta and Dhandayuthapani (2013) : Optimization of Phenol Biodegradation by Pseudomonas pudita Isolated from industrial effluent, Int J Pharm Bio Sci 2013 July; 4(3): (B) 405 - 413

10. Yan, J., Jianping W., Jing, B., Daoquan, W. and Zongding H. (2006): Phenol biodegradation by the yeast Candida tropicalis in the presence of micresol, Biochem. Eng. J., 29, 227-234 